\title{
Filtering Problems for Conditionally Linear Systems with Non-Gaussian Initial Conditions
}

By

\author{
Shigeomi HARA*
}

\begin{abstract}
We solve the filtering problems for conditionally linear systems, that is, stochastic. partially observable systems which are linear in unobservable processes and nonlinear in observable processes, with non-Gaussian initial conditions. We assume that all coefficients of given SDEs are Lipshitz continuous in the observable processes, and that certain quantities concerning the diffusion coefficients of given SDEs are uniformly nonsingular. But we assume nothing about the initial distribution and allow that a part of the coefficients of the given SDEs are of linear growth order in the observable processes.
\end{abstract}

\section{Introduction}

The filtering problems for stochastic, partially observable linear systems with Gaussian initial conditions are solved by Kalman and Bucy [4]. Here "solve" means obtaining the formula to compute the optimal mean square estimate of the present value of the unobservable processes, using the finite dimensional statistics, when we have the data of the past and present observation. In Liptser and Shiryayev [7], they solve the filtering problems for stochastic, partially observable systems that are linear in unobservable processes, and nonlinear in observable processes, that is, conditionally linear systems, with Gaussian initial conditions. On the other hand, in Makowski [8], they solve the filtering problems for stochastic, partially observable linear systems with non-Gaussian initial conditions.

In this paper, we join above two methods in [7] and [8], and solve the filtering problems for conditionally linear systems with non-Gaussian initial conditions. Namely, we consider the following system of stochastic differential equations :

$$
\begin{aligned}
& d X_{t}=\left\{a_{0}(t, Y)+a_{1}\left(t, Y^{Y}\right) X_{t}\right\} d t+b_{1}\left(t, Y^{Y}\right) d W_{1}(t)+b_{2}\left(t, Y^{\prime}\right) d W_{2}(t), \\
& d Y_{t}=\left\{A_{0}(t, Y)+A_{1}(t, Y) X_{t}\right\} d t+B_{1}(t, Y) d W_{1}(t)+B_{2}(t, Y) d W_{2}(t),
\end{aligned}
$$

Communicated by H. Araki, December 25, 1992.

1991 Mathematics Subject Classification: 93E11.

* Department of Applied Mathematics and Physics, Faculty of Engineering, Kyoto University, Kyoto 606, Japan. 
where $X_{t}$ is an unobservable process, $F_{t}$ is an observable process, and $W_{1}, W_{2}$ are mutually independent standard Brownian motions. $X_{0}$ is an arbitrary $\mathscr{F}_{0^{-}}$ measurable random variable, and $Y_{0}=0$. We assume that all coefficients of above SDEs are Lipshitz continuous in the observable processes, and that certain quantities concerning the diffusion coefficients of above SDEs are uniformly nonsingular. In [5], Kolodziej and Mohler study the same problem. They assume the only certain integrabilities for the coefficients of given SDEs. But they also assume a certain integrability of the initial distribution, while we assume nothing about the initial distribution. In [2], Haussman and Pardoux study more general problem that contains also "Beneš problem", which is nonlinear in unobservable processes. There they assume only boundedness of the coefficients of given SDEs, while we allow that a part of coefficients are of linear growth order in the observable processes.

In section 1, we give the precise formulation of the problem and the assumptions, and consider the reduction of the problem to the case $b_{2} \equiv 0$. In section 2 , we prove the main theorem under the assumptions in section 1 . In section 3 , we prove the proposition used in section 1 .

\section{$\S 1$. Problem Formulation and Assumptions}

Let $(\Omega, \mathscr{I}, P)$ be a probability space. In order to guarantee the existence of the regular conditional probability used below, we assume that $(\Omega, \mathscr{F})$ is a standard measurable space (see e.g. [3]). Let $\left(\mathscr{F}_{t}\right)_{t \in[0, T]}(T>0)$ be a right continuous increasing family of sub $\sigma$-fields of $\mathscr{F}$.

The unobservable process $X_{t}$ and the observable process $Y_{t}$ are $M$ and $N$ dimensional $\mathscr{F}_{t}$-adapted continuous processes, respectively, which satisfy the following stochastic differential equations:

$$
\begin{aligned}
& X_{t}=X_{0}+\int_{0}^{t}\left\{a_{0}\left(s, Y^{-}\right)\right.\left.+a_{1}(s, Y) X_{s}\right\} d s+\int_{0}^{t} b_{1}(s, Y) d W_{1}(s) \\
&+\int_{0}^{t} b_{2}(s, Y) d W_{2}(s), \\
& Y_{t}=\int_{0}^{t}\left\{A_{0}(s, Y)+A_{1}(s, Y) X_{s}\right\} d s+\int_{0}^{t} B_{1}(s, Y) d W_{1}(s) \\
&+\int_{0}^{t} B_{2}(s, Y) d W_{2}(s), \quad t \in[0, T],
\end{aligned}
$$

where $W_{1}(t)$ and $W_{2}(t)$ are mutually independent $M$ and $N$ dimensional standard $\mathscr{F}_{t}$-Brownian motions, respectively. $a_{0}, a_{1}, b_{1}, b_{2}, A_{0}, A_{1}, B_{1}$, and $B_{2}$ are progressively measurable mappings from $[0, T] \times C\left([0, T] ; \mathbb{R}^{N}\right)$ to $\mathbb{R}^{M}, \boldsymbol{R}^{M \times M}, \boldsymbol{R}^{M>M}$, $\boldsymbol{R}^{M \times N}, \boldsymbol{R}^{N}, \mathbb{R}^{N \times M}, \mathbb{R}^{N \wedge M}$, and $\boldsymbol{R}^{N \times N}$, respectively. $X_{0}$ is an $\mathbb{R}^{M}$ valued $\mathscr{I}_{0^{-}}$ measurable random variable which is generally non-Gaussian distributed.

We denote by $q_{t}$ the $\sigma$-algebra generated by $\left\{Y_{s}: s \leqq t\right\}$. It is well known 
that if $E\left[\left|X_{t}\right|^{2}\right]<+\infty$, then the optimal mean square estimation of $X_{t}$ from the observation data $y_{t}$ is

$$
E\left[X_{t} \mid y_{t}\right] \text {. }
$$

We aim at computing this using the solutions of finite dimensional stochastic differential equations.

We assume either following Assumptions A or Assumptions B for the coefficients of the SDEs (1.1)-(1.2). We define the $M \times(M+N)$ matrix $b$, and the $N \times(M+N)$ matrix $B$ by

$$
b=\left(\begin{array}{ll}
b_{1} & b_{2}
\end{array}\right), \quad B=\left(\begin{array}{ll}
B_{1} & B_{2}
\end{array}\right) .
$$

Assumptions A. (1) Let $g(t, y)$ be any element of matrices $a_{0}(t, y), a_{1}(t, y)$, $A_{0}(t, y), A_{1}(t, y), b_{1}(t, y), b_{2}(t, y), B_{1}(t, y)$, and $B_{2}(t, y)$. Then, for any $x, y \in$ $C\left([0, T] ; \boldsymbol{R}^{N}\right)$ and $t \in[0, T]$, we have

$$
\begin{aligned}
& |g(t, y)|^{2} \leqq C\left\{\int_{0}^{t}\left(1+\left|y_{s}\right|^{2}\right) d K(s)+\left(1+\left|y_{t}\right|^{2}\right)\right\}, \\
& |g(t, x)-g(t, y)|^{2} \leqq C\left\{\int_{0}^{t}\left|x_{s}-y_{s}\right|^{2} d K(s)+\left|x_{t}-y_{t}\right|^{2}\right\},
\end{aligned}
$$

where $C$ is a constant and $K(s)$ is a nondecreasing right continuous function, $0 \leqq K(s) \leqq 1$.

(2) $a_{1}, A_{1}$ are bounded.

(3) $\exists \delta>0 B B^{*}(t, y) \geqq \delta I$ for any $t \in[0, T], y \in C\left([0, T] ; \boldsymbol{R}^{N}\right)$, where $*$ denotes the transpose of a matrix.

(4) $B_{1}, B_{2}$ are bounded.

(5) $\exists \delta>0$ s.t.

(i) $b b^{*}(t, y) \geqq \delta I$,

(ii) $B\left(I-b^{*}\left(b b^{*}\right)^{-1} b\right) B^{*}(t, y) \geqq \delta I$, for any $t \in[0, T], y \in C\left([0, T] ; \boldsymbol{R}^{N}\right)$.

Assumptions B. (1)-(3) are the same as (1)-(3) of Assumptions A.

(4) $b_{2}(t, y)=0$ for any $t \in[0, T], y \in C\left([0, T] ; \boldsymbol{R}^{N}\right)$.

(5) There exists an $\boldsymbol{R}^{N \backslash M}$ valued bounded progressively measurable function $\tilde{A}_{1}(t, y)$ which satisfies

$$
A_{1}(t, y)=B_{2}(t, y) \tilde{A}_{1}(t, y), \quad t \in[0, T], y \in C\left([0, T] ; \boldsymbol{R}^{N}\right) .
$$

Remark. When $M=N=1$, then (ii) of Assumptions A (5) becomes

$$
\left(b_{1}^{2}+b_{2}^{2}\right)^{-1}\left(B_{1} b_{2}-b_{1} B_{2}\right)^{2} \geqq \delta .
$$

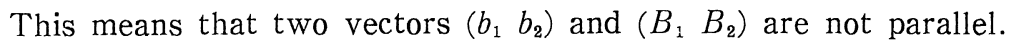

At first, we shall consider the reduction of problem to the case $b_{2}(t, y) \equiv 0$. Under Assumption B, we have nothing to do for this reduction. 
Lemma 1.1 (Lemma 10.4, $[6])$. Let $W(t)(0 \leqq t \leqq T)$ be an $N$ dimensional standard $\mathscr{F}_{t}$-Brownian motion, and $B_{t}$ be an $\boldsymbol{R}^{n \times N}$ valued $\mathscr{F}_{t}$-adapted process such that

$$
\int_{0}^{T} \operatorname{tr}\left(B_{t} B_{t}^{*}\right) d t<+\infty,
$$

where * denotes transpose and $\operatorname{tr}$ denotes trace of square matrices. Let $D_{t}$ be an $\boldsymbol{R}^{n \times k}$ valued $\mathscr{I}_{t}$-adapted process such that

$$
D_{t} D_{t}^{*}=B_{t} B_{t}^{*} \quad \text { for a.e. } t \in[0, T] \text { a.s. . }
$$

Then, there exists a $k$ dimensional standard $\mathscr{F}_{t}$-Brownian motion $U(t)$ such that for any $t \in[0, T]$,

$$
\int_{0}^{t} B_{s} d W(s)=\int_{0}^{t} D_{s} d U(s) \quad \text { a.s. . }
$$

In view of this lemma, we consider the equation:

that is,

$$
\left(\begin{array}{cc}
b_{1} & b_{2} \\
B_{1} & B_{2}
\end{array}\right)\left(\begin{array}{ll}
b_{1}^{*} & B_{1}^{*} \\
b_{2}^{*} & B_{2}^{*}
\end{array}\right)=\left(\begin{array}{cc}
\tilde{b} & 0 \\
\tilde{B}_{1} & \tilde{B}_{2}
\end{array}\right)\left(\begin{array}{cc}
\tilde{b}^{*} & \tilde{B}_{1}^{*} \\
0 & \tilde{B}_{2}^{*}
\end{array}\right),
$$

$$
\begin{gathered}
b b^{*}=b_{1} b_{1}^{*}+b_{2} b_{2}^{*}=\tilde{b} \tilde{b}^{*}, \\
B b^{*}=B_{1} b_{1}^{*}+B_{2} b_{2}^{*}=\tilde{B}_{1} \tilde{b}^{*}, \\
B B^{*}=B_{1} B_{1}^{*}+B_{2} B_{2}^{*}=\tilde{B}_{1} \tilde{B}_{1}^{*}+\tilde{B}_{2} \tilde{B}_{2}^{*}=\tilde{B} \tilde{B}^{*} .
\end{gathered}
$$

Proposition 1.2. Let either Assumptions $A$ or Assumptions $B$ be fulfilled. Then there exist $\boldsymbol{R}^{M \times M}, \boldsymbol{R}^{N \times M}$, and $\boldsymbol{R}^{N \times N}$ valued progressively measurable functions $\tilde{b}(t, y), \tilde{B}_{1}(t, y)$, and $\tilde{B}_{2}(t, y)$, respectively, which satisfy (1.5)-(1.7). Moreover we have the following.

(1) Let $g(t, y)$ be any element of matrices $\tilde{b}(t, y), \tilde{B}_{1}(t, y)$, and $\tilde{B}_{2}(t, y)$. Then $g(t, y)$ satisfies (1.3) and (1.4).

(2) $\exists \delta>0 \widetilde{B} \widetilde{B}^{*}(t, y) \geqq \delta I$ for any $t \in[0, T], y \in C\left([0, T] ; \boldsymbol{R}^{N}\right)$.

(3) There exists an $\boldsymbol{R}^{N \backslash M}$ valued bounded, progressively measurable function $\tilde{A}_{1}(t, y)$ which satisfies

$$
A_{1}(t, y)=\tilde{B}_{2}(t, y) \tilde{A}_{1}(t, y), \quad t \in[0, T], y \in C\left([0, T] ; \boldsymbol{R}^{N}\right) .
$$

We shall prove this proposition in section 3 .

By Lemma 1.1 and Proposition 1.2, there exist $M$ and $N$ dimensional, mutually independent, standard $\mathscr{I}_{t}$-Brownian motions $U(t)$ and $\widetilde{W}_{2}(t)$, respectively, and the stochastic differential equations (1.1) and (1.2) are transformed to:

$$
X_{t}=X_{0}+\int_{0}^{t}\left\{a_{0}(s, Y)+a_{1}(s, Y) X_{s}\right\} d s+\int_{0}^{t} \tilde{b}(s, Y) d U(s)
$$




$$
\begin{aligned}
Y_{t}=\int_{0}^{t}\left\{A_{0}(s, Y)+\right. & \left.A_{1}(s, Y) X_{s}\right\} d s+\int_{0}^{t} \widetilde{B}_{1}(s, Y) d U(s) \\
& +\int_{0}^{t} \widetilde{B}_{2}(s, Y) d \widetilde{W}_{2}(s), \quad t \in[0, T] .
\end{aligned}
$$

\section{§2. Main Theorem}

Following the idea of Makowski [8], we shall introduce the process $x_{t}$ which satisfies an ordinary differential equation:

$$
\frac{d x_{t}}{d t}=a_{1}(t, Y) x_{t}, \quad x_{0}=X_{0},
$$

that is, letting $\Phi(t, Y)$ be the fundamental matrix with $\Phi(0, Y)=I$,

We denote

$$
x_{t}=\Phi(t, Y) X_{0} .
$$

$$
\bar{X}_{t}=X_{t}-x_{t},
$$

then we can rewrite (1.8)-(1.9) to:

$$
\begin{aligned}
\bar{X}_{t}= & \int_{0}^{t}\left\{a_{0}(s, Y)+a_{1}(s, Y) \bar{X}_{s}\right\} d s+\int_{0}^{t} \tilde{b}(s, Y) d U(s) \\
Y_{t}= & \int_{0}^{t}\left\{A_{0}(s, Y)+A_{1}(s, Y) \bar{X}_{s}\right\} d s+\int_{0}^{t} \tilde{B}_{1}(s, Y) d U(s) \\
& +\int_{0}^{t} A_{1}(s, Y) x_{s} d s+\int_{0}^{t} \tilde{B}_{2}(s, Y) d \widetilde{W}_{2}(s), \quad t \in[0, T] .
\end{aligned}
$$

Noting (3) of Proposition 1.2, we set

$$
\begin{aligned}
V_{t} & =\int_{0}^{t} \tilde{A}_{1}(s, Y) x_{s} d s+\widetilde{W}_{2}(t) \\
& =\int_{0}^{t} F(s, Y) X_{0} d s+\widetilde{W}_{2}(t),
\end{aligned}
$$

where,

$$
F(t, y)=\tilde{A}_{1}(t, y) \Phi(t, y)
$$

In order to apply Girsanov's theorem to (2.4), if we set

$$
\Lambda_{t}=\exp \left[-\int_{0}^{\iota}\left\{F(s, Y) X_{0}\right\} * d \widetilde{W}_{2}(s)-\frac{1}{2} \int_{0}^{t}\left|F(s, Y) X_{0}\right|^{2} d s\right], \quad t \in[0, T],
$$

then we get the

Lemma 2.1.

$$
E\left[\Lambda_{T}\right]=1
$$


Proof. Here we follow essentially the argument of Example 2.2 in [2]. Let $p(x, d \omega)$ be the regular conditional probability given $X_{0}=x$. Since $X_{0}$ is $\mathscr{F}_{0}$-measurable, $\widetilde{W}_{2}(t)$ is still an $\mathscr{F}_{t}$-Brownian motion under the probability meas-

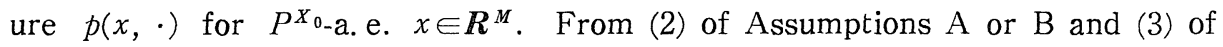
Proposition $1.2, F$ is bounded, so for any $x \in \boldsymbol{R}^{M}$,

$$
\int_{\Omega} \exp \left\{\frac{1}{2} \int_{0}^{T}|F(t, Y(\omega)) x|^{2} d t\right\} p(x, d \omega)<+\infty .
$$

Hence, by Novikov's theorem (see e.g. Theorem III-5.3 in [3]), we have

$$
\int_{\Omega} \Lambda_{T}(\boldsymbol{\omega}) p(x, d \omega)=1, \quad \text { for } P^{x_{0} \text {-a.e. } x \in \boldsymbol{R}^{M} .}
$$

Integrating this equation over $\boldsymbol{R}^{M}$ by $P^{X_{0}}(d x)$, we obtain Lemma 2.1 .

By Lemma 2.1, using Girsanov's theorem, we see that $V_{t}$ is an $\mathscr{F}_{t}$-Brownian motion under the probability measure $\tilde{P}$, where

$$
\tilde{P}(A)=\int_{A} \Lambda_{T}(\omega) P(d \omega), \quad A \in \mathscr{F} .
$$

Lemma 2.2. On the probability space $(\Omega, \mathscr{T}, \tilde{P})$,

(i) $X_{0}, U$, and $V$ are mutually independent;

(ii) $\tilde{P}^{X_{0}}=P^{X_{0}}$ on $\left(\boldsymbol{R}^{M}, \mathscr{B}^{M}\right)$.

Proof. (i) By Girsanov's theorem, $(U(t), V(t))$ is an $M+N$ dimensional $\mathscr{T}_{t^{-}}$ Brownian motion under the probability $\widetilde{P}$. Thus (i) is clear.

(ii) Since $\Lambda_{t}$ is a martingale with respect to $\left(\mathscr{F}_{t}\right)$ under the probability $P$, for any $A \in \mathscr{B}^{M}$ we have

$$
\tilde{E}\left[1_{A}\left(X_{0}\right)\right]=E\left[1_{A}\left(X_{0}\right) \Lambda_{T}\right]=E\left[1_{A}\left(X_{0}\right) \Lambda_{0}\right]=E\left[1_{A}\left(X_{0}\right)\right] .
$$

Now, we have the stochastic differential equations:

$$
\begin{array}{r}
\bar{X}_{t}=\int_{0}^{t}\left\{a_{0}(s, Y)+a_{1}(s, Y) \bar{X}_{s}\right\} d s+\int_{0}^{t} \tilde{b}(s, Y) d U(s) \\
Y_{t}=\int_{0}^{t}\left\{A_{0}(s, Y)+A_{1}(s, Y) \bar{X}_{s}\right\} d s+\int_{0}^{t} \tilde{B}_{1}(s, Y) d U(s) \\
+\int_{0}^{t} \tilde{B}_{2}(s, Y) d V(s), \quad t \in[0, T],
\end{array}
$$

on the probability space $(\Omega, \mathscr{T}, \widetilde{P})$. By (1), (2) of Assumptions A or B and (1) of Proposition 1.2, the SDEs (2.8)-(2.9) have a unique strong solution, so we have

$$
\sigma\left(\bar{X}_{s}, Y_{s} ; 0 \leqq s \leqq t\right) \subset \sigma(U(s), V(s) ; 0 \leqq s \leqq t)
$$

For any $\phi: \boldsymbol{R}^{M} \rightarrow \boldsymbol{R}$, bounded Borel measurable mapping, we have the Kal- 
lianpur-Striebel formula:

$$
E\left[\phi\left(X_{t}\right) \mid a_{t}\right]=\frac{\tilde{E}\left[\phi\left(X_{t}\right) Z_{t} \mid q_{t}\right]}{\tilde{E}\left[Z_{t} \mid q_{t}\right]}, \quad t \in[0, T],
$$

where $\tilde{E}$ denotes the expectation under the probability $\tilde{P}$, and $Z_{t}=\Lambda_{t}^{-1}$, i.e.

$$
Z_{\iota}=\exp \left[X_{0}^{*} \int_{0}^{t} F(s, Y)^{*} d V(s)-\frac{1}{2} X_{0}^{*} \int_{0}^{t}\left(F^{*} F\right)(s, Y) X_{0} d s\right]
$$

We set

$$
h_{t}=\int_{0}^{t} F(s, Y) * d V(s)
$$

and

$$
m_{\iota}=\tilde{E}\left[\left(\begin{array}{c}
\bar{X}_{t} \\
h_{t}
\end{array}\right) \mid a_{t}\right], \quad m_{t}^{\prime}=\tilde{E}\left[\bar{X}_{t} \mid y_{\iota}\right], \quad \gamma_{\iota}=\tilde{E}\left[\left(\left(\begin{array}{c}
\bar{X}_{t} \\
h_{\iota}
\end{array}\right)-m_{\iota}\right)\left(\left(\begin{array}{c}
\bar{X}_{t} \\
h_{\iota}
\end{array}\right)-m_{t}\right) * \mid y_{t}\right] .
$$

Proposition 2.3. Let either Assumptions $A$ or Assumptions $B$ be fulfilled, and $(\Omega, \mathscr{T}, \tilde{P})$ be a probability space. Then, for any $t \in[0, T],\left(\bar{X}_{t}, h_{t}\right)$ is Gaussian with mean vector $m_{t}(\omega)$ and covariance matrix $\gamma_{t}(\omega)$ under the regular condi-

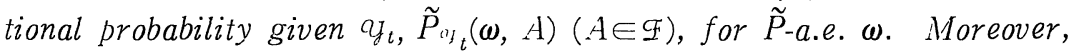

$$
\begin{gathered}
d m_{\iota}=\left(\begin{array}{c}
a_{0}+a_{1} m_{t}^{\prime} \\
0
\end{array}\right) d t+\left\{\left(\begin{array}{c}
\tilde{b} \tilde{B}_{1}^{*} \\
F \tilde{B}_{2}^{*}
\end{array}\right)+\gamma_{t}\left(\begin{array}{c}
A_{1}^{*} \\
0
\end{array}\right)\right\}\left(\tilde{B} \tilde{B}^{*}\right)^{-1} \\
\times\left\{d Y_{t}-\left(A_{0}+A_{1} m_{t}^{\prime}\right) d t\right\}, \quad m_{0}=0, \\
\dot{r}_{t}=\left(\begin{array}{cc}
a_{1} & 0 \\
0 & 0
\end{array}\right) r_{\iota}+r_{\iota}\left(\begin{array}{cc}
a_{1}^{*} & 0 \\
0 & 0
\end{array}\right)+\left(\begin{array}{cc}
\tilde{b} \tilde{b}^{*} & 0 \\
0 & F F^{*}
\end{array}\right)-\left\{\left(\begin{array}{c}
\tilde{b} \tilde{B}_{1}^{*} \\
F \tilde{B}_{2}^{*}
\end{array}\right)\right. \\
\left.+r_{t}\left(\begin{array}{c}
A_{1}^{*} \\
0
\end{array}\right)\right\}\left(\tilde{B} \tilde{B}^{*}\right)^{-1}\left\{\left(\begin{array}{c}
\tilde{b} \tilde{B}_{1}^{*} \\
F \tilde{B}_{2}^{*}
\end{array}\right)+r_{\iota}\left(\begin{array}{c}
A_{1}^{*} \\
0
\end{array}\right)\right\}^{*}, \quad r_{0}=0 .
\end{gathered}
$$

Proof. By assumptions, we can apply Theorem 12.7 of [7] to equations (2.8), (2.9) and (2.12).

We set

$$
g_{t}=\frac{1}{2} X_{0}^{*} \int_{0}^{t}\left(F F^{*}\right)(s, Y) X_{0} d s
$$

Clearly we have

$$
\tilde{E}\left[\phi\left(X_{t}\right) Z_{t} \mid q_{t}\right]=\tilde{E}\left[\tilde{E}\left[\dot{\phi}\left(\bar{X}_{t}+x_{t}\right) \exp \left\{X_{0}^{*} h_{t}-g_{t}\right\} \mid q_{t} \vee \sigma\left(X_{0}\right)\right] \mid q_{t}\right] .
$$

We denote by $\tilde{P}_{0}$ the regular conditional probability given $q_{t} \vee \sigma\left(X_{0}\right)$ on the probability space $(Q, \mathscr{I}, \widetilde{P})$. Since $x_{t}, X_{0}$, and $g_{t}$ are $q_{t} \vee \sigma\left(X_{0}\right)$-measurable, by the property of the conditional expectation we have 


$$
\begin{aligned}
& \check{E}\left[\phi\left(\bar{X}_{t}+x_{t}\right) \exp \left\{X_{0}^{*} h_{t}-g_{t}\right\} \mid \vartheta_{t} \vee \sigma\left(X_{0}\right)\right](\omega) \\
& =\int_{\Omega} \phi\left(\bar{X}_{t}(\tilde{\omega})+x_{t}(\omega)\right) \exp \left\{X_{0}^{*}(\omega) h_{t}(\tilde{\omega})-g_{t}(\omega)\right\} \tilde{P}_{0}(\omega, d \tilde{\omega}), \text { for } \tilde{P}_{\text {-a.e. } \omega .}
\end{aligned}
$$

By (2.10) and (2.12), we have

$$
\sigma\left(\bar{X}_{s}, Y_{s}, h_{s} ; 0 \leqq s \leqq t\right) \subset \sigma(U(s), V(s) ; 0 \leqq s \leqq t) .
$$

Hence, from Lemma 2.2 (i),

$$
(\bar{X}, Y, h) \text { and } X_{0} \text { are mutually independent under } \tilde{P} \text {. }
$$

Therefore we can see that, under the conditional probability $\tilde{P}_{0}(\omega, \cdot),\left(\bar{X}_{t}, h_{t}\right)$ is Gaussian with mean vector $m_{t}(\boldsymbol{\omega})$ and covariance matrix $\gamma_{t}(\boldsymbol{\omega})$ for $\tilde{P}_{\text {-a.e. }} \boldsymbol{\omega}$. We denote by $\mu(d x ; m, \gamma)$ the Gaussian distribution over $\boldsymbol{R}^{2 M}$ with mean vector $m$ and covariance matrix $\gamma$. Thus, from (2.16) we have

$$
\begin{aligned}
& \tilde{E}\left[\boldsymbol{\phi}\left(\bar{X}_{t}+x_{t}\right) \exp \left\{X_{0}^{*} h_{t}-g_{t}\right\} \mid \varphi_{t} \vee \sigma\left(X_{0}\right)\right](\boldsymbol{\omega}) \\
& =\int_{R^{2 N S}} \phi\left(x^{\prime}+x_{t}(\boldsymbol{\omega})\right) \exp \left\{X_{0}^{*}(\boldsymbol{\omega}) x^{\prime \prime}-g_{t}(\boldsymbol{\omega})\right\} \mu\left(d x ; m_{t}(\boldsymbol{\omega}), \gamma_{l}(\boldsymbol{\omega})\right),
\end{aligned}
$$

for $\tilde{P}$-a. e. $\omega$, where $x=\left(\begin{array}{l}x^{\prime} \\ x^{\prime \prime}\end{array}\right), x^{\prime}, x^{\prime \prime} \in \boldsymbol{R}^{M}$. Hence, by (2.15) and (2.18) we have

$$
\begin{aligned}
& \tilde{E}\left[\phi\left(X_{t}\right) Z_{t} \mid y_{t}\right] \\
& \quad=\tilde{E}\left[\int_{R^{2 M}} \phi\left(x^{\prime}+x_{t}\right) \exp \left\{X_{0}^{*} x^{\prime \prime}-g_{t}\right\} \mu\left(d x ; m_{t}, \gamma_{t}\right) \mid y_{t}\right], \quad \tilde{P} \text {-a.s. }
\end{aligned}
$$

Since $X_{0}$ and $a_{t}$ are mutually independent and $m_{t}, \gamma_{t}$ are $a_{t}$-measurable, we have

$$
\begin{gathered}
\tilde{E}\left[\phi\left(X_{t}\right) Z_{t} \mid a_{t}\right](\omega) \\
=\tilde{E}\left[\int_{R^{2 M}} \phi\left(x^{\prime}+\Phi(t, y) X_{0}\right) \exp \left\{X_{0}^{*} x^{\prime \prime}-\frac{1}{2} X_{0}^{*} \int_{0}^{t}\left(F F^{*}\right)(s, y) X_{0} d s\right\}\right. \\
\mu(d x ; m, \gamma)]_{y=Y(\omega), m=m_{t}(\omega), \gamma=\gamma_{t}(\omega)}, \\
=\int_{R^{M}} \int_{R^{2 M}} \phi\left(x^{\prime}+\Phi(t, y) z\right) \exp \left\{z^{*} x^{\prime \prime}-\frac{1}{2} z^{*} \int_{0}^{t}\left(F F^{*}\right)(s, y) z d s\right\} \\
\left.\mu(d x ; m, \gamma) P^{X_{0}}(d z)\right|_{y=Y(\omega), m=m_{t}(\omega), \gamma=\gamma_{t}(\omega),}
\end{gathered}
$$

for $\tilde{P}$-a. e. $\omega$. Here we have used Lemma 2.2 (ii).

Theorem 2.4. Let either Assumptions $A$ or Assumptions $B$ be fulfilled. Then for any $\phi: \boldsymbol{R}^{M} \rightarrow \boldsymbol{R}$, bounded Borel measurable, the optimal mean square estimation $E\left[\phi\left(X_{t}\right) \mid y_{t}\right]$ satisfies Kallianpur-Striebel formula (2.11), and we have 


$$
\begin{aligned}
& \tilde{E}\left[\phi\left(X_{t}\right) Z_{\iota} \mid q_{t}\right](\omega) \\
& =\int_{R^{M}} \int_{R^{2} M} \phi\left(x^{\prime}+\Phi(t, y) z\right) \exp \left\{z^{*} x^{\prime \prime}-\frac{1}{2} z^{*} \int_{0}^{t}\left(F F^{*}\right)(s, y) z d s\right\} \\
& \left.\mu(d x ; m, \gamma) P^{X_{0}}(d z)\right|_{y=Y(\omega), m=m_{t}(\omega), \gamma=\gamma_{t}(\omega)} \text {, for } P \text {-a.e. } \omega,
\end{aligned}
$$

where $x=\left(\begin{array}{l}x^{\prime} \\ x^{\prime \prime}\end{array}\right), x^{\prime}, x^{\prime \prime} \in \boldsymbol{R}^{M}$. Further, $m_{t}(\boldsymbol{\omega})$ and $\gamma_{i}(\boldsymbol{\omega})$ satisfy the stochastic differential equations (2.13) and (2.14).

\section{§3. Proof of Proposition 1.2}

Under Assumptions B, if we set

$$
\tilde{b}=b_{1}, \quad \tilde{B}_{1}=B_{1}, \quad \tilde{B}_{2}=B_{2},
$$

then the conclusion of Proposition 1.2 holds clearly.

Next, we prove the conclusion of Proposition 1.2 under Assumptions A. If we set

$$
\begin{gathered}
\tilde{b}(t, y)=\left(b b^{*}\right)^{1 / 2}(t, y) ; \\
\tilde{B}_{1}(t, y)=B b^{*}\left(b b^{*}\right)^{-1 / 2}(t, y) ; \\
\tilde{B}_{2}(t, y)=\left\{B\left(I-b^{*}\left(b b^{*}\right)^{-1} b\right) B^{*}\right\}^{1 / 2}(t, y), \quad t \in[0, T], y \in C\left([0, T] ; \boldsymbol{R}^{N}\right),
\end{gathered}
$$

then (1.5)-(1.7) are satisfied. By (i) of Assumptions A (5), $\left(b b^{*}\right)^{-1}$ is well defined. (2) of Proposition 1.2 clearly holds by (3) of Assumptions A and (1.7). For (3) of Proposition 1.2, we set

$$
\tilde{A}_{1}(t, y)=\tilde{B}_{2}^{-1} A_{1}(t, y) .
$$

By (ii) of Assumptions A (5), $\tilde{B}_{2}^{-1}$ is well defined, and

$$
\left|\tilde{B}_{2}^{-1}(t, y)\right| \leqq C \delta^{-1 / 2} .
$$

Hence, noting $A_{1}$ is also bounded, we can see that (3) of Proposition 1.2 holds. At last we shall prove (1) of Proposition 1.2. It is clear that

$$
|\tilde{b}| \leqq C\left(\left|b_{1}\right|+\left|b_{2}\right|\right),
$$

so $\tilde{b}$ satisfies (1.3).

Lemma 3.1. Let $b: \boldsymbol{R} \rightarrow \boldsymbol{R}^{n \times m}(n \leqq m)$ satisfy the following:

$$
\begin{gathered}
|b(t)-b(s)| \leqq K|t-s|, \quad t, s \in \boldsymbol{R} ; \\
b b^{*}>0 .
\end{gathered}
$$

Then if we set $a=b b^{*}$, we have

$$
\left|a^{1 / 2}(t)-a^{1 / 2}(s)\right| \leqq C K|t-s|, \quad t, s \in \boldsymbol{R},
$$


where the constant $C$ depends only on $n$ and $m$.

Proof. We follow essentially the proof of Theorem 5.2.2 in [9], i.e., Lemma 3.2 in this paper.

At first we consider the case $b(t) \in C^{1}(\boldsymbol{R})$. Then clearly $a(t) \in C^{1}(\boldsymbol{R})$. By Lemma 6.1.1 in $[1]$, we have also $a^{1 / 2}(t) \in C^{1}(R)$. It is sufficient to prove that

$$
\sup _{t \in R}\left|\left(a^{1 / 2}\right)^{\prime}(t)\right| \leqq C K .
$$

Here' denotes the differential in $t$. Let any $t \in \mathbb{R}$ be fixed. We may assume that $a(t)$ is a diagonal matrix. Indeed, there exists an orthogonal matrix $Q$ such that $\tilde{a}(t) \equiv Q a(t) Q^{*}$ is diagonal. Clearly $\tilde{a}^{1,2}(t)=Q a^{1 / 2}(t) Q^{*}$. Therefore

$$
\left|\left(\tilde{a}^{1 / 2}\right)^{\prime}(t)\right|=\left|\left(a^{1 / 2}\right)^{\prime}(t)\right| .
$$

So let $a(t)$ be diagonal. Since $a^{1 / 2}(t)$ is also diagonal, we have

$$
a^{\prime}(t)_{\imath j}=\left(a^{1 / 2}\right)^{\prime}(t)_{\imath \jmath} a^{1 / 2}(t)_{\jmath \jmath}+a^{1 / 2}(t)_{\imath \imath}\left(a^{1 / 2}\right)^{\prime}(t)_{\imath \jmath} .
$$

Here the suffix $i j$ denotes the $(i, j)$ component of matrices. Hence

$$
\left(a^{1 / 2}\right)^{\prime}(t)_{\imath \jmath}=\frac{a^{\prime}(t)_{\imath j}}{a^{1 / 2}(t)_{\imath \imath}+a^{1 / 2}(t)_{\jmath \jmath}} .
$$

On the other hand,

$$
a^{\prime}(t)_{\imath j}=\sum_{k=1}^{m}\left\{b^{\prime}(t)_{2 k} b(t)_{j k}+b(t)_{\imath k} b^{\prime}(t)_{j k}\right\} .
$$

So,

$$
\left|a^{\prime}(t)_{{ }_{2 j}}\right| \leqq K \sum_{k=1}^{m}\left\{\left|b(t)_{j_{k}}\right|+\left|b(t)_{{ }_{2}}\right|\right\} .
$$

Since $a(t)$ is diagonal, we have

$$
a^{1 / 2}(t)_{\imath \imath}=\sqrt{a(t)_{\imath \imath}}=\sqrt{\sum_{k=1}^{m} b(t)_{\imath k}^{2}} .
$$

Hence,

$$
\left|\left(a^{1 / 2}\right)^{\prime}(t)_{\imath \jmath}\right| \leqq \frac{K \sum_{k=1}^{m}\left\{\left|b(t)_{j k}\right|+\left|b(t)_{\imath k}\right|\right\}}{\sqrt{\sum_{k=1}^{m} b(t)_{\imath_{k}}^{2}}+\sqrt{ } \sum^{\prime} \sum_{k=1}^{m} b(t)_{j k}^{2}} \leqq m K
$$

and we obtain (3.6).

When $b(t) \notin C^{1}(\boldsymbol{R})$, it is sufficient that we approximate $b(t)$ by $b_{\varepsilon}(t)$ with the molifier.

By this lemma, we can directly prove that $\tilde{b}$ satisfies (1.4). So we see that $\tilde{b}$ satisfies (1) of Proposition 1.2.

In view of the definition of $\tilde{B}_{1}$, say $(3.2)$, we set

$$
4=\left(b b^{*}\right)^{-1 / 2} b \text {. }
$$


Since

$$
A A^{*}=\left(b b^{*}\right)^{-1 / 2} b b^{*}\left(b b^{*}\right)^{-1 / 2}=I
$$

we have

$$
|A|^{2} \leqq C_{1}\left|A . A^{*}\right| \leqq C_{1}
$$

Hence

$$
\left|\widetilde{B}_{1}\right|=\left|B_{1} A^{*}\right| \leqq C_{2}|B| \cdot\left|A^{*}\right| \leqq C_{3}|B| \leqq C_{1}\left(\left|B_{1}\right|+\left|B_{2}\right|\right) .
$$

Therefore $\tilde{B}_{1}$ is bounded, and satisfies (1.3). From (1.6), we have

$$
\begin{aligned}
\tilde{B}_{1}(x)- & \tilde{B}_{1}(y)=-\hat{B}_{1}(y)(\tilde{b}(x)-\tilde{b}(y)) \tilde{b}(x)^{-1} \\
& +\sum_{i=1}^{2}\left\{\left(B_{\imath}(x)-B_{\imath}(y)\right) b_{\imath}^{*}(x) \tilde{b}^{-1}(x)+B_{\imath}(y)\left(b_{\imath}^{*}(x)-b_{\imath}^{*}(y)\right) \tilde{b}^{-1}(x)\right\},
\end{aligned}
$$

for $x, y \in C\left([0, T] ; \boldsymbol{R}^{N}\right)$. Here we abbreviated the argument $t . B_{1}, B_{2}$ and $\tilde{B}_{1}$ are bounded. Clearly $b_{\imath}^{*} \tilde{r}^{-1}=b_{l}^{*}\left(b b^{*}\right)^{-1 / 2}(\imath=1,2)$ is bounded. By (i) of Assumptions $\mathrm{A}(5), \tilde{b}^{-1}$ is bounded. Hence from (3.7) we can see that $\tilde{B}_{1}$ satisfies (1.4). So $\tilde{B}_{1}$ satisfies (1) of Proposition 1.2 .

From (1.7), using the boundedness of $B_{1}, B_{2}$ and $\tilde{B}_{1}$, we can see that $\tilde{B}_{2}$ is also bounded. So $\tilde{B}_{2}$ satisfies (1.3). By the definition of $A$, we have

$$
A(x)-A(y)=\tilde{b}(y)^{-1}\{(b(x)-b(y))-(\tilde{b}(x)-\tilde{b}(y))+A(x)\}
$$

By the boundedness of $b^{-1}$ and $A, A$ satisfies (1.4). Since $B$ and $A$ is bounded and satisfy (1.4), so is $B\left(I-A^{*} A\right) B^{*}=\widetilde{B}_{2}^{2}$.

Lemma 3.2 (Theorem 5.2.2, [9]). Let $a_{(}^{(}()$be a symmetric matrix valued function of $t \in \boldsymbol{R}$, which satisfies

$$
\begin{aligned}
& |a(t)-a(s)| \leqq K|t-s| \quad t, s \in \boldsymbol{R} ; \\
& \exists \delta>0 \quad a(t) \geqq \delta I \quad \text { for any } t \in \boldsymbol{R} .
\end{aligned}
$$

Then we have

$$
\left|a^{1 / 2}(t)-a^{1 / 2}(s)\right| \leqq C K|t-s|
$$

where $C$ is a constant.

By (ii) of Assumptions A (5), using this lemma, we can see that $\hat{B}_{2}$ satisfies (1.4).

This completes the proof of Proposition 1.2.

\section{References}

[1] Friedman, A., Stochastic differential equations and applications, 1, Academic Press, New York, 1975.

[2] Haussmann, U.G. and Pardoux, E., A conditionally almost linear filtering problem with non-Gaussian initial condition, Stochastics. 23 (1988), 241-275.

[3] Ikeda, N. and Watanabe. S., Stochastic differential equations and difjusion proc- 
esses, North Holland/Kodansha, Amsterdam/Tokyo, 1981.

[4] Kalman, R. E. and Bucy, R.S., New results in linear filtering and prediction theory, Trans. ASME J. Basic Engrg., 83D (1961), 95-108.

[5] Kolodziej, W. J. and Mohler, R. R., State estimation and control of conditionally linear systems, SIAM J. Control and Optimization. 24 (1986), 497-508.

[6] Liptser, R.S. and Shiryayev, A.N., Statistics of Random Processes I-General Theory, Springer-Verlag, New York, 1977.

[7] Liptser, R.S. and Shiryayev, A. N., Statistics of Random Processes II-Applications, Springer-Verlag, New York, 1978.

[8] Makowski, A.M., Filtering formulae for partially observed linear systems with non-Gaussian initial conditions, Stochastics, 16 (1986), 1-24.

[9] Strook, D. W. and Varadhan, S. R. S., Multidimensional diffusion processes, SpringerVerlag, New York, 1979. 